\title{
The nature of the prompt X-ray and radio emission from SN 2002ap ${ }^{\star}$
}

\author{
F. K. Sutaria ${ }^{1}$, P. Chandra ${ }^{2,3}$, S. Bhatnagar ${ }^{4,5}$, and A. Ray ${ }^{2}$ \\ ${ }^{1}$ Department of Physics and Astronomy, The Open University, Milton Keynes, UK \\ 2 Tata Institute of Fundamental Research, Bombay 400 005, India \\ 3 Joint Astronomy Programme, Indian Institute of Science, Bangalore 560 012, India \\ 4 National Centre for Radio Astrophysics, Pune 411 007, India \\ 5 National Radio Astronomy Observatory, Socorro, NM 87801, USA \\ Received 3 July 2002 / Accepted 30 October 2002
}

\begin{abstract}
We report on the combined X-ray and radio observations of the type Ic SN 2002ap, using XMM-Newton TOO observation of M 74 and the Giant Metrewave Radio Telescope (GMRT). We account for the presence of a nearby source in the pre-supernova Chandra field of view in our measurements of the X-ray flux $(0.3-10 \mathrm{KeV}) 5.2$ days after the explosion. The X-ray spectrum is well fitted by a power law spectrum with photon index $\alpha=2.6$. Our results suggest that the prompt $\mathrm{X}$-ray emission originates from inverse Compton scattering of photospheric thermal emission by energetic electrons. Radio observations with the GMRT at $610 \mathrm{MHz}$ (8 days after the explosion) and $1420 \mathrm{MHz}$ (70 days after the explosion) are combined with the high frequency VLA observations of SN 2002ap reported by Berger et al. (2002), and the early radiospheric properties of SN 2002ap are compared with similar data from two other supernovae. Finally, the GMRT radio map reveals four other $\mathrm{X}$-ray sources in the field of view of M 74 with radio counterparts.
\end{abstract}

Key words. supernovae: individual: SN 2002ap - supernovae: general - radiation mechanisms: non-thermal circumstellar matter

\section{Introduction}

Supernovae are known to be explosions of massive and intermediate mass stars, although the nature of the progenitor star for different supernova types, remains an area of longstanding research. Currently, it is believed that type II and type Ib or Ic supernovae arise from core collapse of massive stars, while the more homogeneous type Ia supernovae are the results of thermonuclear explosions. There is a considerable variety among the core collapse supernovae spectroscopically and in their kinetic energy. The "hypernova" class among these are believed to have explosion energy significantly in excess of $10^{51} \mathrm{erg}$, found in "normal" supernovae. The spatial and temporal near-coincidence of type Ic SN 1998bw with the long duration GRB980425 has provided further impetus to observational and theoretical studies of type $\mathrm{Ib} / \mathrm{Ic} \mathrm{SNe}$ and their progenitors (MacFadyen et al. 2001; Meszaros et al. 1993).

During a supernova explosion the interaction of the outer parts of the stellar ejecta with the circumstellar matter gives rise to a high energy density shell. X-ray emission is expected from both shocked circumstellar matter and the shocked supernova matter (see e.g. Chevalier 1982; Chevalier \& Fransson 2001).

Send offprint requests to: F. K. Sutaria,

e-mail: F.K.Sutaria@open.ac.uk

* Based on observations made with XMM-Newton Observatory and the Giant Metre Wave Radio Telescope.
In addition, the interaction region may also accelerate electrons to relativistic energies and amplify pre-existing magnetic fields which gives rise to nonthermal synchrotron emission seen in many supernovae. The radio and the X-ray emission give information on a region of the supernova which may be far removed from the optical photosphere (which has a smaller radius), although the conditions in the optical photosphere may determine the X-ray emission characteristics in some instances. In general, X-ray and radio observations in early stages of a supernova can be used to determine (1) the total mass lost from the pre-supernova star before explosion and (2) constrain various physical processes leading to X-ray and radio emission.

The type Ic SN 2002ap was discovered on Jan. 29.4, 2002 (Y. Hirose as reported by Nakano et al. 2002) in NGC 628 (M 74), at a distance of only 7.3 Mpc. Based on spectral analysis of the early observations, the epoch of explosion was estimated at Jan. $28.0 \pm 0.52002$ UT (Mazzali et al. 2002). For the purpose of the analysis presented in this paper, we will regard Jan. 28.0 as the date of explosion. The broad spectral features (Kinugasa et al. 2002; Meikle et al. 2002; Gal-Yam et al. 2002), and a subsequent modeling of its spectroscopic and photometric data (Mazzali et al. 2002), suggested that this was an energetic event with explosion energy $E \simeq 4-10 \times 10^{51} \mathrm{erg}$. In this paper, we discuss our analysis of the XMM-Newton observation in the X-ray (0.1-15 keV) bands (Sect. 2), accounting for the presence of a nearby object in the pre-supernova 
X-ray field, observed earlier by the Chandra X-ray Observatory (Sect. 2.1). We also observed SN 2002ap in the $0.61 \mathrm{GHz}$ and $1.4 \mathrm{GHz}$ radio bands (see Sect. 3), and the implications of the GMRT upper limits in the context of VLA observations at the same epoch (Berger et al. 2002) are discussed in Sect. 4.

We also summarize the explosion parameters that we derived from the optical observations and modeling reported by Mazzali et al. (2002), as this is used as input for later sections (Sect. 4). We have also combined the GMRT data with the VLA data (Berger et al. 2002) to derive conditions near the radiosphere. A combined analysis of the early X-ray and radio observations is presented in Sect. 5, which attempts to constrain the multiple physical processes (thermal and non-thermal) that are responsible for the early X-ray emission. We also compare SN 2002ap with 2 other SNe (1998bw (Ic) and 1993J (IIb) for which early multi frequency data is available. Finally, in Sect. 6 we discuss these results in the context of the presupernova star and its evolution.

\section{X-ray observations and data analysis}

SN 2002ap and its host galaxy M 74 (NGC 628) were observed by XMM-Newton, four days after the discovery of the SN. Rodriguez Pascual et al. (2002) and Soria \& Kong (2002) have reported the EPIC-PN detection of the supernova, as well as several other sources in the host galaxy. Here we present a spectral analysis of the XMM-Newton EPIC-PN and -MOS CCD detection, taking into account contributions from other pre-existing sources present in the extraction circle, near the supernova.

XMM-Newton observed the field of view of SN 2002ap in the full-frame, thin filter mode, for the EPIC-PN and the two EPIC-MOS cameras. Simultaneously, it also observed M 74 with the Optical Monitor in the UVW1. The full exposure in the EPIC CCDs was $~ 37.4$ ks. Since Chandra X-ray observatory also observed the same field on June 19, 2001 and October 19, 2001 for a total of $47 \mathrm{ks}$, we present our analysis, based on both the presupernova and post-supernova exposures.

Early analysis of the XMM-Newton TOO observations of SN 2002ap showed that the supernova is rather faint in X-rays with a flux of $\sim 10^{-14} \mathrm{erg} \mathrm{cm}^{-2} \mathrm{~s}^{-1}$. Because the psf of the XMM EPIC-CCDs is rather large ( $\sim 50^{\prime \prime}$ to enclose $90 \%$ of the energy at $10 \mathrm{keV}$ ), it was necessary to verify the absence of, or to account for the presence of any other sources, however faint, within the spectral extraction region on the EPIC CCDs.

\subsection{Chandra observations of the presupernova field}

In order to check if there were any other sources present in the vicinity of the optical position of SN 2002ap (RA = $01^{\mathrm{h}} 36^{\mathrm{m}} 23^{\mathrm{s}} .85$, Dec $=+15^{\circ} 45^{\prime} 13^{\prime \prime} .2$ ), we examined the archival Chandra observations of M 74, carried out on 19th Oct. 2001. In the net $46.2 \mathrm{ksec}$ of exposure time with Chandra-ACIS, we did not see any source at the position of the supernova. However, we did find a bright source at RA $01^{\mathrm{h}} 36^{\mathrm{m}} 23^{\mathrm{s}} .404$ and Dec $+15^{\circ} 44^{\prime} 59^{\prime}$. 89 , which, being only $14.9^{\prime \prime}$ away from the $\mathrm{SNe}$, was well within the region of XMM spectral extraction. Comparing the positions of bright, point-like sources seen in both the Chandra and XMM fields of view, we found that the relative astrometric shift between Chandra and $\mathrm{XMM}$ is at most $\sim 5^{\prime \prime}$. Thus, this object (hereafter, CXU J013623.4+154459) would have been detected within the 40" circle used to extract the XMM-EPIC spectra.

Level-2 pipeline processed Chandra data was used for spectral analysis, since a check on the calibration files used in the archived level-2 processed data showed that no improvement would be made by re-calibrating the data. The spectrum was extracted using the CIAO software, and the spectral analysis was carried out using the SHERPA software. The source was located on ACIS-S6 chip, and a light curve was extracted from a source free region of the ACIS-S6 chip to check that the observation was not contaminated by background flares. We also extracted a light curve for the source, to check for flaring, or any periodic variation, but the object is too faint for any such variability to be noted. Since no sources are seen within $10^{\prime \prime}$ of this object, we used a region of radius 6 " to extract the spectrum. As a measure of the PSF at the source location, a radial region of $\simeq 1.5^{\prime \prime}$ would enclose $50 \%$ of the energy at $1.49 \mathrm{keV}$.

CXU J013623.4+154459 is very faint, and the higher energy $(E \geq 9 \mathrm{keV})$ spectrum is seen to be strongly dominated by the background - possibly due to high energy particles, rather than X-ray events. Because of the problems associated with accurately modeling the high energy component of the background spectra, we used background subtracted data and restricted our analysis to the energy range $0.3-10.0 \mathrm{keV}$. The background subtracted count rate in this energy band is $6.5( \pm 2.4) \times 10^{-4} \mathrm{~s}^{-1}$. Fixing the column density at $0.49 \times 10^{21} \mathrm{~cm}^{2}$, (obtained from online hydrogen column density generator of High Energy Astrophysics Archive (HEASARC), in the direction of the SN) and using the $\chi^{2}$ Gehrels statistic, we found that the source was well fitted by a power-law spectrum with a photon index $0.6_{-0.35}^{+0.6}$, implying a $0.3-10.0 \mathrm{keV}$ flux of $1.3 \times 10^{-14} \mathrm{ergs} \mathrm{cm}^{-2} \mathrm{~s}^{-1}$. The $\chi^{2}$ goodness-of-fit parameter was $Q=0.899$, where $Q$ measures the probability of obtaining the observed (or larger) value of $\chi^{2}$, if the data was well represented by the fitted parameters. For an ideal fit, $Q=1$.

\subsection{XMM-Newton observations of SN 2002ap field}

SN 2002ap was observed by the XMM instruments from Feb. 2.03 to Feb. 2.42 UT, 2002 (estimated 5 days after the explosion), for a duration of $37.4 \mathrm{ksec}$. Both EPIC-MOS and EPIC-PN observations were carried out in the "Thin1" filter mode. The data was pipeline processed and calibrated using the XMM-Science Analysis Software (SAS) version 5.2, and the latest available versions of the calibration files. The events in the EPIC-MOS1, EPIC-MOS2 and EPIC-PN data sets were filtered using the SAS-xmmselect task, using the appropriate flags and event selection criteria to account for event pile-up. We followed the analysis procedure recommended in "Users Guide to the XMM-Newton Science Analysis System" (2001), with the exception of allowing patterns $\leq 4$ in the case of EPIC$\mathrm{PN}$, to account for any pile-up, however negligible it may be. A time series analysis of the entire EPIC-PN, MOS1 and MOS2 


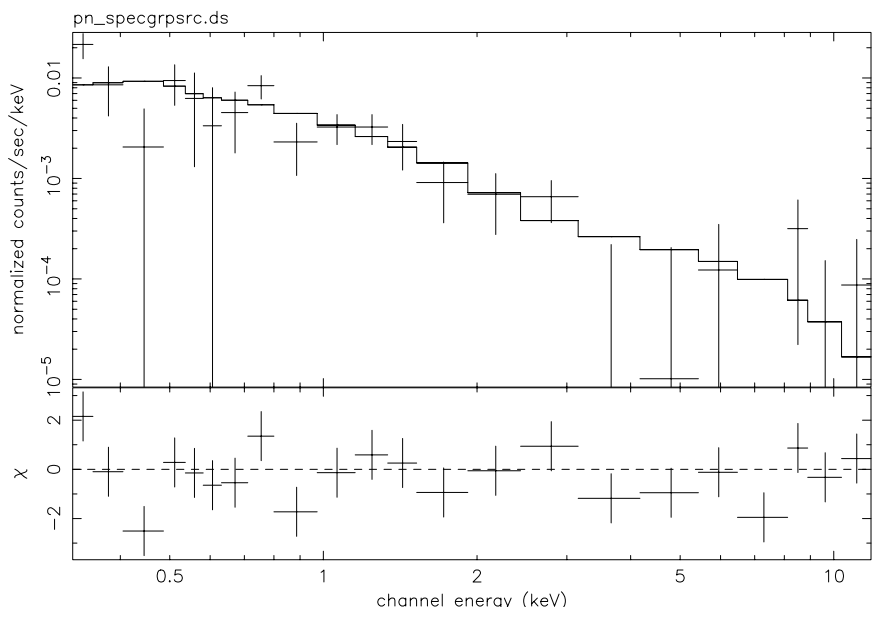

Fig. 1. EPIC-PN spectrum and the fitted power law model (see Sect. 2.3) of SN 2002ap. The model fitted here consists of an absorbed powerlaw of photon index $=-2.6$ with an additional constant absorbed power law component to account for CXU J013623.4+154459. $\log N_{\mathrm{H}}$ was fixed at 20.68. The residuals are plotted in the lower panel.

data, shows that there are times of large, random fluctuation over the entire field of view of both EPIC-PN and MOS CCDs. Filtering out these intervals of highly fluctuating background reduced the exposure time to $25.5 \mathrm{ks}$ in EPIC-PN and $\sim 30 \mathrm{ks}$ in each of EPIC-MOS1 and MOS2.

SN 2002ap was identified on the EPIC-CCDs using the optical coordinates $\left(\mathrm{RA}=01^{\mathrm{h}} 36^{\mathrm{m}} 23^{\mathrm{s}} .85\right.$, Dec $\left.=+15^{\circ} 45^{\prime} 13^{\prime \prime} .2\right)$. The EPIC-PN spectra was extracted using a circle of radius $40^{\prime \prime}$, within which almost $80 \%$ of the source photons in the energy range $10.5-1.5 \mathrm{keV}$ would be enclosed, with the extraction region remaining on a single CCD chip. The EPICMOS1 and EPIC-MOS2 spectra were extracted using a psf of radius 50 ", which encloses $90 \%$ of the total energy. The response matrices and ancillary response files were generated using the SAS-rmfgen and SAS-arfgen tasks, and the appropriate line number for the PN-CCD. The data was grouped and analysed using the XSPEC software.

\subsection{Results of $X$-ray observations}

The $0.3-12 \mathrm{keV}$, background subtracted, count rate in XMM EPIC-PN is $6.6( \pm 1.0) \times 10^{-3} \mathrm{~s}^{-1}$. In the same energy range, the EPIC-MOS1 count rate is $1.69( \pm 0.64) \times 10^{-3} \mathrm{~s}^{-1}$ and the EPIC-MOS2 count rate is $1.7( \pm 0.64) \times 10^{-3} \mathrm{~s}^{-1}$.

In order to account for the presence of the Chandra source discussed above in the XMM spectral extraction region, all models fitted to the data had an additive absorbed power-law component, with parameters derived from fits to the Chandra data, as discussed above. Since the $\mathrm{SN}$ is very faint, and the spectra rather sparse (Fig. 1), we kept $N_{\mathrm{H}}=0.049 \times 10^{22} \mathrm{~cm}^{-2}$ as constant for all fits. In general, we found that letting $N_{\mathrm{H}}$ vary can often result in unphysically low values of $N_{\mathrm{H}} \ll$ $10^{-16} \mathrm{~cm}^{-2}$, while providing little improvement in the value of reduced $\chi^{2}$. In Table 1, we have quoted the parameters from the best fitted models in our analysis - the $0.3-10 \mathrm{keV}$ flux quoted there is corrected for the presence of the Chandra source. The
Table 1. Best fit spectral parameters for EPIC-PN. $k T$ represents the plasma temperature or the black body temperature, depending on the model. All uncertainties are quoted in $90 \%$ confidence limit. $N_{\mathrm{H}}$ was constant for all fits. The absorbed flux for SN 2002ap is quoted here.

\begin{tabular}{cccccc}
\hline \hline Model & $N_{\mathrm{H}}$ & $\alpha$ & $k T$ & $\chi_{v}^{2} /$ d.o.f. & $\left.f\right|_{0.3-10}$ \\
\hline & $10^{21} \mathrm{~cm}^{-2}$ & & $\mathrm{keV}$ & & $10^{-14} \mathrm{erg} \mathrm{cm}^{-2} \mathrm{~s}^{-1}$ \\
\hline Power-law & 0.49 & $2.6_{0.5}^{0.6}$ & - & $1.2 / 20$ & 1.07 \\
& 0.42 & $2.5_{0.5}^{0.6}$ & - & $1.2 / 20$ & 1.0 \\
Thermal & 0.49 & - & $0.84_{-0.3}^{+0.9}$ & $1.2 / 20$ & 0.81 \\
Brems. & & & & & \\
Raymond- & 0.49 & - & $2.31_{-0.8}^{+1.9}$ & $1.58 / 20$ & 1.04 \\
$\quad$ Smith & & & & & \\
Blackbody & 0.49 & - & $0.21_{-.06}^{+0.1}$ & $1.4 / 20$ & 0.6 \\
\hline
\end{tabular}

data is rather sparse, hence $\chi^{2}$-fitting is unable to distinguish between thermal bremsstrahlung and the simple power law models. The power law distribution (Fig. 1) fits the data well with $\alpha=2.6$, as does the thermal bremsstrahlung model with temperature $k T \sim 0.8 \mathrm{keV}$. Using the powerlaw model, we find that the total flux in the EPIC-PN extraction region is $2.4 \times 10^{-14} \mathrm{ergs} \mathrm{cm}^{-2} \mathrm{~s}^{-1}$. Correcting for the presence of CXU $\mathrm{J} 013623.4+154459$, the $0.3-10.0 \mathrm{keV}$ flux from SN 2002ap is $1.07_{-0.31}^{+0.63} \times 10^{-14} \mathrm{ergs} \mathrm{cm}^{-2} \mathrm{~s}^{-1}$.

Adding an extra power-law, or a cutoff power-law component to the bremsstrahlung, or Raymond-Smith or blackbody models did not improve the fit, and resulted in poorly constrained values of temperatures with an unusually high photon index $\alpha \sim 9.0$, and hence these models were discarded.

Because of the low count rate in EPIC-MOS CCDs, it was decided to fit to the combined MOS1 and MOS2 data, allowing only the relative normalisations to vary. As discussed for EPIC-PN data, each model incorporates an absorbed power-law component to account for the Chandra source. Thus, the data was well fitted by an absorbed thermal bremsstrahlung, with $\chi^{2} /$ d.o.f. $=0.95 / 19$ and $k T=0.4_{-0.22}^{1.28}$. The $0.3-10 \mathrm{keV}$ flux (corrected for CXU J013623.4+154459) was $7.1 \times 10^{-15} \mathrm{ergs} \mathrm{cm}^{-2} \mathrm{~s}^{-1}$.

SN 2002ap was too faint to be detected by either of the RGS detectors.

\subsection{The Optical Monitor observation}

The XMM-Newton Optical Monitor observed SN 2002ap in the 4-frame (ENG-2) mode with $U V W 1$ filter for a duration of $2.5 \mathrm{ks}$. The $U V W 1$ filter peaks at $\lambda \simeq 270 \mathrm{~nm}$, and spans the range $\sim 250 \leq \lambda \leq \sim 310 \mathrm{~nm}$. The frames were combined and the data was pipeline processed using SAS version 5.3 with the latest available calibration files. A source list was compiled. We find that the optical monitor count rate for SN 2002ap was $14.67( \pm 0.08) \mathrm{s}^{-1}$, with the background count rate being $0.8 \mathrm{~s}^{-1}$. This corresponds to a $U V W 1$ flux of $7.667( \pm 0.002) \times 10^{-15} \mathrm{erg} \mathrm{cm}^{-2} \mathrm{~s}^{-1} \AA^{-1}$. 


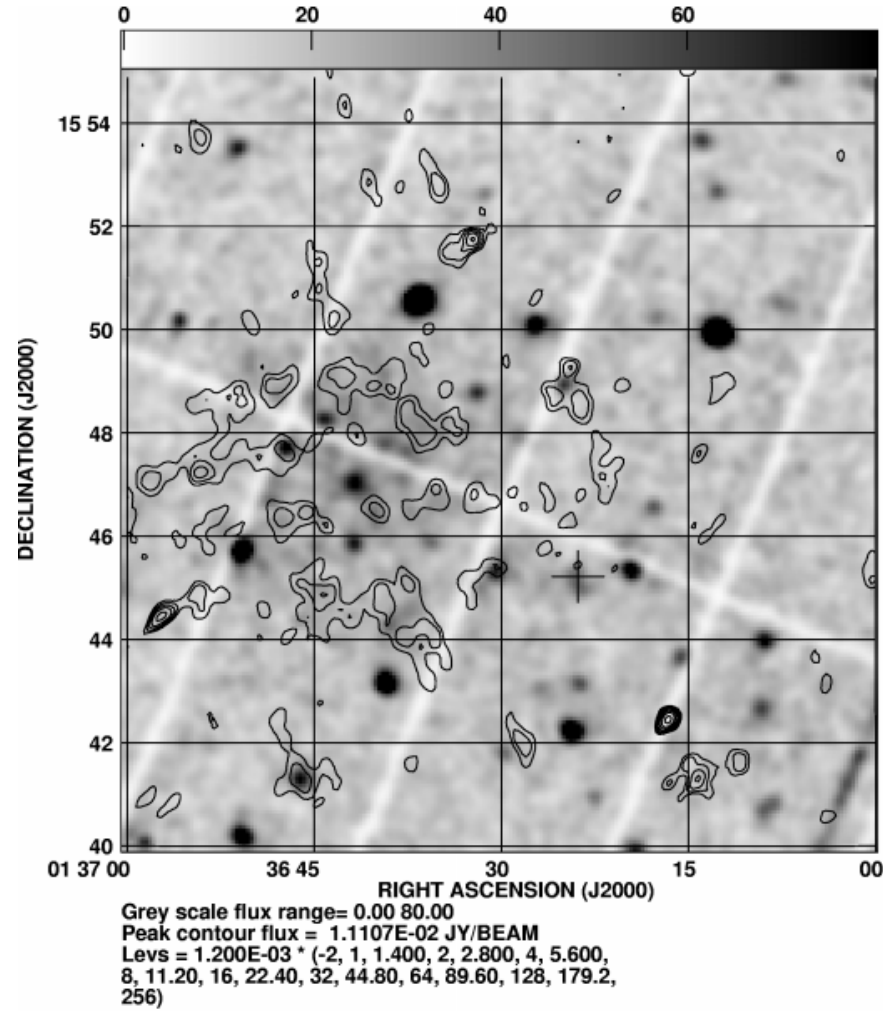

Fig. 2. GMRT image of the field at $610 \mathrm{MHz}$ containing SN 2002ap overlayed on the X-Ray image from XMM. X-ray image is on a grey scale while the radio image is the contour map with lowest level of $1.2 \mathrm{mJy}$. The image resolution is $15^{\prime \prime} \times 15^{\prime \prime}$. Four X-ray sources having radio counterparts are seen in this figure. They are numbered according to their decreasing RA in Table 4. Position of the SN on the map is marked with a cross.

\section{GMRT observations of SN 2002ap field}

The radio observations of SN 2002ap were made with the Giant Metrewave Radio Telescope in aperture synthesis mode on 2002 February 5.56 in the $610 \mathrm{MHz}$ band and on 2002 April 8.22 in the $1420 \mathrm{MHz}$ band.

For both the observations at 610 as well as $1420 \mathrm{MHz}$, the pointing centre was chosen to be the position of supernova i.e. $\mathrm{RA}=01^{\mathrm{h}} 36^{\mathrm{m}} 23^{\mathrm{s}} .85$ and $\mathrm{Dec}=15^{\circ} 45^{\prime} 13^{\prime \prime}$. 2 . In the $610 \mathrm{MHz}$ observations the time on source was around 2 hours and $3 \mathrm{C} 48$ was used as the flux, phase and the bandpass calibrator. It was also used as bandpass calibrator. 3C48 was observed for approximately $10 \mathrm{~min}$ after every $30 \mathrm{~min}$. It's flux at $610 \mathrm{MHz}$ was derived from the best fit spectra given in VLA calibrator manual. Out of a total of 30 antennas, we had 22 good antennas. The full band of $16 \mathrm{MHz}$ was used, resulting in 128 frequency channels of width $\sim 125 \mathrm{kHz}$. For the observations in $1420 \mathrm{MHz}$ band, the number of antennas used were 24 . The time on the source was $\sim 3.5$ hours. 3C48 was again used as a flux calibrator and it was observed at the beginning and at the end of the observations. The flux of 3C48 was assumed to be $16.15 \mathrm{Jy}$ from VLA calibrator manual. Compact sources $0119+321$ and $0238+166$ were used as the phase calibrator with fluxes $2.6 \mathrm{Jy}$ and $1.26 \mathrm{Jy}$ respectively. 3C48 was also used
Table 2. GMRT observations for flux and phase calibrators.

\begin{tabular}{cccccc}
\hline \hline Source & Date in & RA & Dec. & $v$ & Flux \\
& 2002 & J2000 & J2000 & MHz & Jy \\
\hline 3C48 & 5 Feb. & 013741.3 & 330935 & 610 & 29.4 \\
3C48 & 8 Apr. & 013741.3 & 330935 & 1420 & 16.2 \\
$0119+321$ & 8 Apr. & 011935.0 & 321050 & 1420 & 2.9 \\
$0238+166$ & 8 Apr. & 023838.9 & 163659 & 1420 & 1.0 \\
\hline
\end{tabular}

Table 3. Results of GMRT observations of SN 2002ap.

\begin{tabular}{ccccc}
\hline \hline $\begin{array}{c}\text { Date in } \\
2002\end{array}$ & $\begin{array}{c}v \\
(\mathrm{MHz})\end{array}$ & $\begin{array}{c}\text { Resolution } \\
(\operatorname{arcsec})\end{array}$ & $\begin{array}{c}2 \sigma \text { Flux } \\
(\mathrm{mJy})\end{array}$ & $\begin{array}{c}\text { rms } \\
\text { mJy/ beam }\end{array}$ \\
\hline 5 Feb. & 610 & $9.5 \times 6$ & $<0.34$ & 0.17 \\
8 Apr. & 1420 & $8 \times 3$ & $<0.18$ & 0.09 \\
\hline
\end{tabular}

as a bandpass calibrator. The full band of $16 \mathrm{MHz}$ resulting in 128 channels of width $\sim 125 \mathrm{kHz}$ was used.

\subsection{Data reduction, calibration and results}

The data was analysed using AIPS (Astronomical Image Processing Software) of National Radio Astronomy Observatory. Interference in some of the frequency channels, spikes and dropouts due to electronics etc. were flagged using the AIPS task FLGIT. FLGIT was run on the calibrator scans and 10 times the expected noise per channel was used as the flagging criterion. In this manner $15 \%$ of the data was flagged. Then one clean channel was looked at and antenna and baseline based flagging was done. Approximately $20 \%$ of the data was lost in the flagging process. After computing the single channel antenna-based complex gains for the flux calibrator and phase calibrator with the time resolution of $20 \mathrm{~s}$, bandpass calibration was done using 3C48 to apply the single channel calibration to all the channels. To get rid of the effect of the band smearing at low frequency $(610 \mathrm{MHz})$, a pseudocontinuum data base of 6 frequency channels was made from the central 100 channels of $16 \mathrm{MHz}$ bandwidth. Field maps were made by Fourier transformation and cleaning. Iterative self-calibration rounds were run for phase correction. Details of GMRT data analysis procedures are given in (Bhatnagar 2000).

In neither of the observations carried out on 2002 February 5 (day 8.56) and on 2002 April 8 (day 42), SN 2002ap was detected by GMRT. The upper limits of the fluxes from the region of the $\mathrm{SN}$ are given in Table 3. The GMRT $610 \mathrm{MHz}$ contour map shows several extended sources, which are likely associated with the spiral arms of the galaxy. Superposition of the $610 \mathrm{MHz}$ contour map on the X-ray EPIC-PN image (Fig. 2), reveals the existence of $4 \mathrm{X}$-ray sources with radio counterparts. These are listed in Table 4 with their radio flux densities and X-ray $0.3-10.0 \mathrm{keV}$ count rates. Spectral analysis to determine the nature of these sources is being done separately.

\section{The location of the radiosphere}

The radio turn-on of supernovae are found to be wavelength dependent, with the flux at shorter wavelengths peaking before 
Table 4. List of X-ray sources with radio counterparts in M 74.

\begin{tabular}{|c|c|c|c|c|}
\hline $\begin{array}{c}\text { Source } \\
\text { No. }\end{array}$ & $\begin{array}{c}\text { RA } \\
\text { J2000 }\end{array}$ & J2000 & $\begin{array}{l}\text { Radio } \\
\text { Flux } \\
(\mathrm{mJy})\end{array}$ & $\begin{array}{l}\text { EPIC-PN } \\
\text { count-rate } \\
10^{-3} \text { ct s }^{-1}\end{array}$ \\
\hline 1 & 013647.2 & 154745 & $7.1( \pm 1.3)$ & $4.3( \pm 1.3)$ \\
\hline 2 & 013646.1 & 154117 & $12.8( \pm 1.5)$ & $3.9( \pm 1.3)$ \\
\hline 3 & 013624.9 & 154858 & $22.7( \pm 1.5)$ & $1.9( \pm 1.3)$ \\
\hline 4 & 013630.5 & 154517 & $4.5( \pm 1.3)$ & $4.6( \pm 1.3)$ \\
\hline
\end{tabular}

Table 5. Optical photosphere of SN 2002ap on day 5 (Feb. 2, 2002). The distance modulus was $\mu=29.5$, and the maximum value of bolometric correction was used (Allen 1976).

\begin{tabular}{cccccc}
\hline \hline$M_{v}$ & $M_{\text {bol }}$ & $\begin{array}{c}T_{\text {eff }} \\
\mathrm{K}\end{array}$ & $\begin{array}{c}R_{\text {opt }} \\
\mathrm{cm}\end{array}$ & $\begin{array}{c}\bar{v}_{\mathrm{ph}} \\
\mathrm{km} \mathrm{s}^{-1}\end{array}$ & $\begin{array}{c}F_{\text {UVOIR }} \\
\mathrm{erg} \mathrm{cm}^{-2} \mathrm{~s}^{-1}\end{array}$ \\
\hline-17.4 & -16.5 & 11000 & $3.4 \times 10^{14}$ & 8000 & $2 \times 10^{-10}$ \\
\hline
\end{tabular}

that at longer wavelengths (e.g. Berger et al. 2002). This decreasing absorption can be either via free-free absorption (FFA) or synchrotron self-absorption (SSA) in the expanding CSM. We have fitted the VLA and GMRT data on 2002 Feb. 5.96 to a SSA model, with spectral index $=-0.8$ in the optically thin limit, implying that the radius of the radio photosphere on this day was $R_{\mathrm{r}}=3.5 \times 10^{15} \mathrm{~cm}$ and the magnetic field in the shocked ejecta was $B=0.29 \mathrm{G}$ (see Table 6 ). We also note that that SSA prediction of flux at $610 \mathrm{MHz}$ band is consistent with the GMRT upper limit.

In order to compare the photospheric radii across wavebands, we also compute the size of the optical photosphere at the same epoch. Using the synthetic light curve best-fitted to the bolometric light curve on day 5 (Mazzali et al. 2002), the bolometric magnitude was $M_{\mathrm{bol}}=-16.5$, and the visual magnitude was $M_{\mathrm{v}}=-17.1$ (using a distance modulus of 29.5 Sharina et al. 1996). This implies that the radius of the optical photosphere on day 5 was $R_{\mathrm{opt}}=3.4 \times 10^{14} \mathrm{~cm}$, and corresponding total flux across UV, optical and IR bands was $F_{\text {UVOIR }}=2 \times 10^{-10} \mathrm{erg} \mathrm{cm}^{-2} \mathrm{~s}^{-1}$. The inferred conditions in the optical photosphere are listed in Table 5.

In contrast, the position of the radio photosphere on day 7 was $r=4.5 \times 10^{15} \mathrm{~cm}$ and its velocity $90000 \mathrm{~km} \mathrm{~s}^{-1}$ (Berger et al. 2002). Scaling to day $5, R_{\text {radio }} \sim 3.2 \times 10^{15} \mathrm{~cm}$. Thus, the radio photosphere is quite far outside the optical photosphere. The region of production of the X-rays is also outside the optical photosphere radius (see Sect. 6). This substantiates the inference from the early spectrum (Meikle et al. 2002) that there was sufficient material at $v \geq 30000 \mathrm{~km} \mathrm{~s}^{-1}$.

The framework of spherical symmetry has been used to perform the above analysis. This is a reasonable assumption on the day of the XMM observation and shortly afterwards, since the month-long, early spectropolarimetric data of Wang et al. (2002) implied there was little distortion of the photosphere on 2002 February 3.

Finally, we compare the early radiospheric properties of SN 2002ap with those of other radio SNe, across type classification, using equipartition arguments. Theoretically, early radio emission from $\mathrm{SNe}$ is thought to originate from an

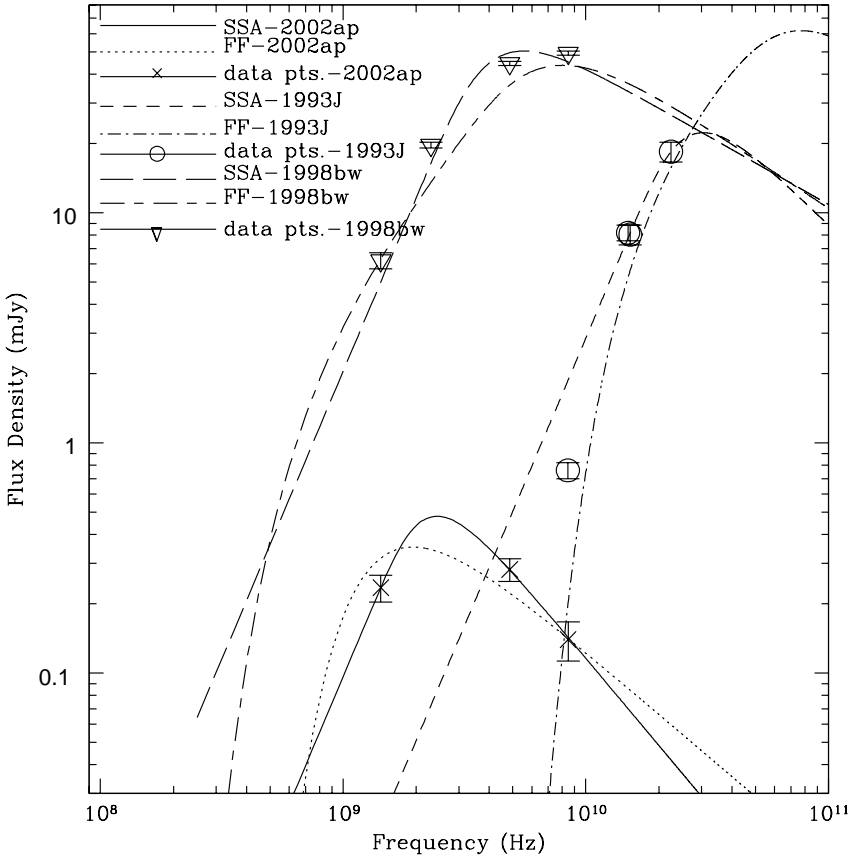

Fig. 3. Comparison of spectrum of three SNe (SN1993J van Dyk et al. 1994; SN 1998bw Kulkarni et al. 1998; SN 2002ap Berger et al. 2002) near day 11 after explosion. Solid line, dashed line and long dashed lines show the best fit synchrotron self absorption model for SN 2002ap, 1993J and 1998bw respectively and dotted line, dotdashed line and short-long dashed line show the corresponding free free absorption model.

Table 6. Best fit parameters of SSA for SN 2002ap on day 8.96 i.e. Feb. 5.96, 2002.

\begin{tabular}{ccccc}
\hline \hline$\alpha$ & $v_{\mathrm{p}}$ & $F_{\mathrm{p}}$ & $R_{\mathrm{r}}$ & $B$ \\
& $\mathrm{GHz}$ & $\mu \mathrm{Jy}$ & $\mathrm{cm}$ & $\mathrm{G}$ \\
\hline 0.8 & 2.45 & 397 & $3.5 \times 10^{15}$ & 0.29 \\
\hline
\end{tabular}

envelope that is expanding at a substantially higher speed than the optical photosphere (Shklovsky 1985). It is only at early times after the explosion that the effect of Compton boosting by electrons to high energy bands producing nonthermal spectrum is potentially observable (Sect. 6). At such early times radio spectra of supernovae are relatively rare.

We have fitted models (Fig. 3) to the radio spectra obtained from three supernovae: SN 1993J (a type IIb SN) on day 11.45, SN 1998bw (a type Ic SN) on day 11.7 and SN 2002ap (another type Ic $\mathrm{SN}$ ) on day 11.0. Best fit parameter values for SSA model were obtained by using a $\chi^{2}$ fit. While SN 1993J and SN 1998bw were much brighter at their peaks compared to SN 2002ap, the synchrotron self-absorption peak for SN 2002ap seems to occur at the lowest frequency among all three. Best fit parameters values are listed in Table 7. The equipartition (angular) radius (that gives the minimum energy density in combined magnetic and relativistic particle energy densities) obtained by Slysh (1963):

$\theta_{\mathrm{eq}}=120 d_{\mathrm{Mpc}}^{-1 / 17} S_{\mathrm{p}, \mathrm{mJy}}^{8 / 17} v_{\mathrm{p}, \mathrm{GHz}}^{(-2 \alpha-35) / 34} \mu \mathrm{as}$ 
Table 7. SN 1993J, SN 1998bw and SN 2002ap best fit parameters of SSA on day 11.

\begin{tabular}{ccccccc}
\hline \hline SNe & $v_{\mathrm{p}}$ & $F_{\mathrm{p}}$ & $\Theta_{\mathrm{eq}}$ & $\begin{array}{c}U_{\mathrm{eq}} \\
\times 10^{45} \\
\mathrm{erg}\end{array}$ & $\begin{array}{c}B_{0} \\
\mathrm{G}\end{array}$ & $\begin{array}{c}R_{0} \\
\times 10^{15} \\
\mathrm{~cm}\end{array}$ \\
\hline 2002ap & 2.45 & 0.48 & 39.0 & 0.69 & 0.47 & 4.80 \\
1998bw & 5.5 & 50.4 & 112.4 & 3500 & 0.23 & 68.4 \\
1993J & 30.5 & 22.3 & 17.6 & 0.50 & 3.54 & 1.08 \\
\hline
\end{tabular}

appears to be the largest for SN 1998bw (112 $\mu$ as) while those for SN 1993J and SN 2002ap are comparable (18 and $39 \mu$ as respectively). Hence SN 1998bw which was not only coincident with a GRB source but also very bright in the radio was rapidly expanding. It was unusual compared to the more ordinary SN like SN 2002ap (despite both supernova's identical type classification), - the latter having similarity with SN 1993J in terms of equipartition size, across type classification.

\section{Radio and X-ray turn-on and progenitor mass loss}

The early emission in X-ray and Radio bands allows us to estimate the density of the CSM along line of sight, and hence the mass loss rate from the presupernova star, if the terminal wind speed of the progenitor star is known or estimated.

The CSM density is related to the mass loss parameters as: $\rho_{\mathrm{cs}}=\dot{M} / 4 \pi u_{\mathrm{w}} r^{2}$. The gas overlying the emission regions would itself serve as an absorber of the radio and X-ray radiation. An estimate of $\dot{M}$ can be made from the XMM data. The mass loss rate is a measure of the column depth $\int n \mathrm{~d} r$ rather than the $\int n^{2} \mathrm{~d} r$ which appears in the radio (free-free) absorption. The stellar wind would absorb the X-rays below $8 \mathrm{keV}$ due to the dominant photo-electric absorption of the metals. The time at which $\tau \sim 1$ is reached for photons of energy $E_{\mathrm{keV}}$ is (Chevalier \& Fransson 1994):

$t_{\mathrm{X}}=C_{5} \dot{M}_{-5} u_{\mathrm{w} 1}^{-1} u_{4}^{-1} E_{\mathrm{keV}}^{-8 / 3}$.

The value of the constant $C_{5}(n)$ depends upon the density profile $(n)$ of the ejecta $\left(\rho_{\text {ej }}=\rho_{0}\left(t / t_{0}\right)^{-3}(u t / r)^{n}\right)$. Using a typical value of the profile index $n=10.2$, the epoch of X-ray detection as day 5 and $E_{\mathrm{keV}}=0.7$, we determine $\dot{M}_{-5} / v_{\mathrm{w} 1} \leq$ $6.5 \times 10^{-2}$. From optical spectral measurements, the velocity of the pre-supernova wind was $\simeq 580 \mathrm{~km} \mathrm{~s}^{-1}$ (Leonard et al. 2002). This implies that $\dot{M} \leq 4 \times 10^{-5} M_{\odot}$. The above expression assumes that the heavy elements contributing to the opacity are mostly neutral - this upper limit would be less stringent if oxygen in the undisturbed circumstellar medium is heavily ionised, in which case the X-ray absorption is reduced.

A similar mass loss rate can also be derived from the first radio detection of SN 2002ap in the $1.4 \mathrm{GHz}$ band on 2002 Feb. 1.93 (i.e. on day 5) when the optical depth in the radio frequency band would have been $\sim 1$. At this time the dominant opacity source for radio radiation is the free-free opacity from fully ionised wind (Chevalier 1982): from the radio data, we estimate the upper limit on the mass loss rate as $\dot{M} \leq 6 \times 10^{-5} M_{\odot} \mathrm{yr}^{-1}$.

\section{Radiative processes in the early SN}

Our spectral modeling of XMM observation of SN 2002ap shows that both power-law and thermal bremsstrahlung models give acceptable fits to early X-ray data. Since multiwaveband data from UV, optical and radio are also available from this supernova, these can be additionally utilised to restrict models of prompt emission. Among the possible mechanisms invoked in the context of radio loud X-ray supernovae are: a) direct synchrotron emission from radio to X-ray band, b) free-free emission from the hot gas consisting of swept up mass behind the blast wave (circumstellar) shock and that behind the reverse shock, and c) inverse Compton scattering of optical photons emitted by the supernova by the hot electrons.

Case (a): as in the case of SN 1980K (Canizares et al. 1982), the direct synchrotron radiation mechanism for SN 2002ap prompt emission can be eliminated. The power law spectrum in frequency for the radio flux observed from SN 2002ap is: $F_{v}=3 \mathrm{mJy}(3.2 \mathrm{GHz} / v)^{0.9}(t / \text { day) })^{-0.9}$ (unabsorbed flux from Berger et al. 2002). If the same ambient magnetic field ( $B \sim B_{\text {eq }} \sim 0.2$ Gauss) implied by equipartition arguments were to hold, to generate X-ray energies (1 keV) by synchrotron mechanism, would imply the presence of highly relativistic electrons $\left(\gamma \geq 1.4 \times 10^{6}\right)$. On day 5, a straight extrapolation of the radio spectrum with the above flux would imply a flux of only 58 picoJansky at $1 \mathrm{keV}$. Since the $90 \%$ energy bandwidth for a power law distribution of energy index -1.6 in the XMM band is $3.7 \mathrm{keV}$, this would imply an X-ray flux of about $5 \times 10^{-16} \mathrm{erg} \mathrm{s}^{-1} \mathrm{~cm}^{-2}$. This is far less than what is observed by XMM. Thus a direct radio to X-ray synchrotron radiation mechanism is unviable, or produces an insignificant part of the observed X-rays. Therefore, different populations of electrons appear to be responsible for the radio and X-ray fluxes that are seen.

Case (b): the circumstellar gas is heated to high temperatures $(\geq 100 \mathrm{keV})$ by the shock resulting from the piston-like expanding supernova envelope. This shocked gas cools by freefree emission and/or Compton cooling. The free-free luminosity from the circumstellar (blast-wave) and reverse shocks (Fransson 1982; Chevalier \& Fransson 2001) is:

$L_{\mathrm{i}}=3 \times 10^{39} g_{\mathrm{ff}} C_{n}\left(\frac{\dot{M}_{-5}}{u_{\mathrm{w} 1}}\right)^{2}\left(\frac{t}{10 \text { day }}\right)^{-1} \mathrm{erg} \mathrm{s}^{-1}$

where for the reverse shock, $C_{n}=(n-3)(n-4)^{2} / 4(n-2)=$ 7.875 for $n=10$ and $C_{n}=1$ for the circumstellar shock. $g_{\mathrm{ff}}$ is the Gaunt factor.

With $\dot{M}=3.36 \times 10^{-5} M_{\odot} \mathrm{yr}^{-1}$ and with a wind velocity $u_{\mathrm{w}}=1000 \mathrm{~km} \mathrm{~s}^{-1}$ (i.e. $u_{\mathrm{w} 1}=100$ ), at $t=5$ days, the X-ray flux at $7.3 \mathrm{Mpc}$ would be: $8.2 \times 10^{-15} \mathrm{erg} \mathrm{cm}^{-2} \mathrm{~s}^{-1}$. This flux would have been near the observed flux that has been detected from SN 2002ap by XMM. However, with the reported high ejecta velocities and the implied temperature of the shocked ejecta and CSM along with the limited cool absorbing shell at this early stage, one would expect a flat tail of high energy photons up to about $100 \mathrm{keV}$ (Fransson 1982). Since the XMM spectrum on day 5 is not so hard (see Table 1 where the thermal bremsstrahlung temperature is quite modest), the free free emission is also an insignificant part of the early radiative 
Table 8. Comptonizing plasma properties at $t=5 \mathrm{~d}$ with $\gamma=3$ and $R_{\text {opt }}=3.4 \times 10^{14} \mathrm{~cm}$.

\begin{tabular}{ccccc}
\hline \hline Scenario & $\begin{array}{c}\dot{M}_{-5} \\
10^{-5} M_{\odot} / \mathrm{yr}\end{array}$ & $\begin{array}{c}u_{\mathrm{w} 1} \\
10 \mathrm{~km} \mathrm{~s}^{-1}\end{array}$ & $\begin{array}{c}\tau_{\mathrm{e}} \\
T_{\mathrm{e}} \\
10^{9} \mathrm{~K}\end{array}$ \\
\hline Wolf-Rayet & 1.5 & 58 & $4 \times 10^{-4}$ & 2 \\
& 3 & 100 & $4.4 \times 10^{-4}$ & 2 \\
\hline Interacting & 10 & 58 & $2.5 \times 10^{-3}$ & 1.5 \\
Binary & & & & \\
Case-BB & 10 & 10 & $1.5 \times 10^{-2}$ & 1.1 \\
\hline
\end{tabular}

budget. Thermal X-ray emission (i.e. bremsstrahlung) will however become more dominant compared to the inverse Compton process (case $\mathrm{c}$ below) as the supernova ages.

Case (c): the dominant cooling and radiative mechanism at early stages when the optical photospheric temperature $T_{\text {eff }} \geq$ $10^{4} \mathrm{~K}$, is Compton cooling (Fransson 1982). Optical photons from the photosphere can undergo repeated scattering off the hot electrons in the shocked region (notably the shocked CSM) and a power law photon distribution (in energy) can result even if the electron distribution is not a power law, and even if the electron scattering depth $\tau_{\mathrm{e}}$ remains small. Pozdnyakov et al. (1977) reported Monte Carlo calculations and their analytical approximations of the emergent Compton scattered spectrum. The spectrum depends on the optical depth $\tau_{\mathrm{e}}$ and the temperature $T$ of the electron plasma. For photons undergoing electron scattering with cross-section $\sigma_{T}$ in the shocked gas is:

$\tau_{\mathrm{e}}=\frac{\dot{M} \sigma_{\mathrm{T}}}{4 \pi m_{\mathrm{p}} R_{\mathrm{s}} u_{\mathrm{w}}}\left(1-\frac{R_{\mathrm{opt}}}{R_{\mathrm{s}}}\right)$.

The flux density and the energy index $\gamma$ originating from Comptonization are (Fransson 1982):

$\mathcal{F}_{v}^{\text {Compton }} \sim \tau_{\mathrm{e}} \mathcal{F}_{v}^{\text {opt }}\left(v_{\mathrm{o}} / v\right)^{\gamma} \mathrm{erg} \mathrm{s}^{-1} \mathrm{~cm}^{-2} \mathrm{~Hz}^{-1}$

with

$\gamma(\gamma+3)=-\frac{m_{\mathrm{e}} c^{2}}{k T_{\mathrm{e}}} \ln \left[\frac{\tau_{\mathrm{e}}}{2}\left(0.9228-\ln \tau_{\mathrm{e}}\right)\right]$

Here, $v_{\mathrm{O}}$ is the peak frequency in the (optical) input spectrum, and $F_{\mathrm{o}}$ the optical flux density. $R_{\mathrm{opt}}$ is the radius of photosphere. $R_{\mathrm{S}}$ is the position of the plasma element.

We note that on day 5 the unabsorbed (or dereddened) $\mathrm{X}$-ray and optical flux densities derived from XMM and ground based observations (Sect. 4) imply an optical to X-ray power law of $\gamma=2.5-2.8$ and a logarithm of the ratio of flux densities of $\approx 7.4$. The optical to X-ray power law index is consistent with the XMM results (see Table 1). We determine the optical depth and temperature conditions of the Comptonizing plasma for $\gamma=3$ in Table 8 . The presupernova scenarios typical for type Ic SNe, for which the optical depths are reported in Table 8 are discussed in the next section. The plasma has the maximum optical depth at twice the optical photosphere radius. The latter was taken as $3.4 \times 10^{14} \mathrm{~cm}$. Since most of the X-ray emission would take place at $\sim \tau_{\max }$, the relevant plasma outflows with a velocity of approximately $16000 \mathrm{~km} \mathrm{~s}^{-1}$.

\section{Discussion and conclusion}

In this paper, we presented an analysis of the XMM data of the SN 2002ap field and have obtained spectral model fits to the prompt X-ray emission. We compare the X-ray image with the GMRT $610 \mathrm{MHz}$ radio image obtained three days apart. While we find no radio counterpart of the $\mathrm{SN}$ at such low frequencies, several sources in the field have radio and X-ray counterparts.

We compare the radio data obtained from three different supernovae in their early phases and model these using the synchrotron self absorption model. SN 1998bw(Ic) with a GRB counterpart had very different radiosphere radius and equipartition angular sizes at approximately the same time in their evolution compared to two other SNe: SN 2002ap(Ic) and SN 1993J(IIb).

We model the early X-ray emission with inputs from optical photometry and light curve and find that the inverse Compton scattering of optical photons from the supernova photosphere by hot electron plasma can account for the observed early $\mathrm{X}$-ray flux and the spectrum for modest electron temperatures and optical depths. Thermal processes are inefficient initially and would be important only as the supernova ages.

Mass loss rates and stellar wind velocities of the progenitor stars determine the optical depth of shock heated matter due to electron scattering - a key parameter in the production of the X-ray flux from the lower energy photons. These in turn depend upon the scenario and progenitor configuration giving rise to type Ic SNe, e.g.:

1. Massive Wolf-Rayet (WR) stars which have lost their hydrogen and helium envelope before the explosion (Langer et al. 1999) have an empirically determined mass loss rate of 1.5-3.2 $\times 10^{-5} M_{\odot}$ (after taking into account effects due to clumpy medium), (Hamann et al. 1998; Willis 1998) and terminal wind velocities $\sim 1000 \mathrm{~km} \mathrm{~s}^{-1}$ depending upon the type of the WR star.

2. Interacting binaries - in particular, in a case BB mass transfer from a helium star overflowing its Roche-lobe, to a companion removes most of the helium rich layers before the type Ic SN. Habets (1985) finds that a $2.5 M_{\odot}$ helium star during carbon shell burning stage expands to a red giant dimension of $18 R_{\odot}$ and sustains an average mass transfer rate of $10^{-4} M_{\odot} \mathrm{yr}^{-1}$ lasting about 3000 years. During this time, the terminal wind speed of the mass losing star would be typically $100 \mathrm{~km} \mathrm{~s}^{-1}$.

Using the relevant parameters in the above two scenarios, we derive electron optical depth encountered by the intermediate energy photons as listed in Table 8. It is evident that both ranges of electron optical depths for the Compton boosting process remain viable alternatives. For the case of the interacting binary model where the optical depths are somewhat larger, the implied electron temperatures required for the plasma would be lower than in the single star WR model. Such temperatures are well within the range expected for hot circumstellar gas $\left(T_{\mathrm{cs}} \geq 3.6 \times 10^{9} \mathrm{~K}\right)$ even for modest velocities of $16000-20000 \mathrm{~km} \mathrm{~s}^{-1}$ for the hot electron plasma moving above the optical photosphere. 
Acknowledgements. This work made use of XMM Target of Opportunity data. F. K. Sutaria would like to thank the XMM-Newton helpdesk for many useful communications during the pipeline processing of raw data. We acknowledge the use of Chandra archival data on M 74. We thank the staff of the GMRT that made the radio observations possible. We also thank the NRAO staff for providing the Astronomical Image Processing Software (AIPS) for radio data analysis.

\section{References}

Allen, C. W. 1976, Astrophysical Quantities

Berger, E., Kulkarni, S., \& Chevalier, R. 2002, ApJ, 177, L5

Bhatnagar, S. 2000, MNRAS, 317, 453

Canizares, C., Kriss, G., \& Feigelson, E., ApJ, 253, L17

Chevalier, R. A. 1982, ApJ, 259, 302

Chevalier, R. A., \& Fransson, C. 1994, ApJ, 420, 268

Chevalier, R. A., \& Fransson, C. 2001, in Supernovae and Gamma-ray Bursts, ed. K. W. Weiler (Springer-Verlag) [astro-ph/0110060]

Fransson, C. 1982, A\&A, 111, 140

Gal-Yam, A., Ofek, E., \& Shemmer, O. 2002, MNRAS, 332, L73

Gondoin, Ph. 2001, Users guide of the XMM-Newton Science Analysis System, XMM-Newton Science Ops. center

Habets, G. 1985, Ph.D. Thesis, University of Amsterdam

Hamann, W.-R., \& Koesterke, L. 1998, A\&A, 335, 1003

Kinugasa, K., et al. 2002, IAUC, 7811
Kulkarni, S., Frail, D., Wieringa, M. H., et al. 1998, Nature, 395, 663

Langer, N., \& Heger, N. 1999, Proc. IAU Symp. 193, ed. K. van der Hucht, G. Koenigsberger, \& P. R. J. Eenens (Astronomical Society of the Pacific)

Leonard, D. C., Filippenko, A. V., Chornock, R., \& Foley, R. J. 2002, PASP, 114, L1333

Mazzali, P., et al. 2002, ApJ, 572, L61

Meikle, P., et al. 2002, IAUC, 7811

MacFadyen, A., Woosley, S., \& Heger, A. 2001, ApJ, 550, 410

Meszaros, P., \& Rees, M. 1993, ApJ, 405, 278

Nakano, S., Sano, Y., Kushida, R., et al. 2002, IAUC, 7810

Pozdnyakov, L., Sobol, I., \& Sunyaev, R. 1977, Sov. Astron., 21, 708

Rodriguez Pascual, P., Gonzalez Riestra, R., Gonzalez Garcia, B., et al. 2002, IAUC, 7821

Sharina, M. E., Karachentsev, I. D., \& Tikhonov, N. A. 1996, A\&AS, 119,499

Shklovskii, I. S. 1985, Soviet Astron. Lett., 11, 105

Slysh, V. I. 1963, Nature, 199, 682

Soria, R., \& Kong, A. K. H., 2002, ApJ, 572, L33

van Dyk, S. D., Weiler, K. W., Sramek, R. A., Rupen, M. P., \& Panagia, N. 1994, ApJ, 432, L115

Wang, L., Hoeflich, P., Wheeler, J. C., Fransson, C., \& Lundqvist, P. 2002, ApJ, submitted [astro-ph/0206386]

Willis, A. J. 1999, in Proc. IAU Symp. 193, ed. K. van der Hucht, G. Koenisgberger, \& P. R. J. Eenens (Astronomical Society of the Pacific) 